\title{
Multiple Primary Lung Tumors of Different Pathological Types Including Squamous Cell Carcinoma, Adenocarcinoma, and Mixed Squamous Cell and Glandular Papilloma: A Case Report
}

\author{
Xiaojing Wang \\ Haifeng Liu \\ Dongjie Zhai \\ Yanan Qin \\ Chuifeng Fan (D) \\ Di Zhang
}

Department of Pathology, The First Affiliated Hospital and College of Basic Medical Sciences of China Medical University, Shenyang, II000I, People's Republic of China
Correspondence: Di Zhang; Chuifeng Fan Email zhangdi_new@hotmail.com; cffan@cmu.edu.cn

\begin{abstract}
Multiple primary lung cancer (MPLC) is considered relatively rare. This report presents an unusual case of multiple pulmonary nodules in a 74-year-old man who presented with three independent synchronous tumors in the right upper lobe. The tumors were diagnosed as peripheral squamous cell carcinoma (SCC), adenocarcinoma, and pulmonary mixed squamous cell and glandular papilloma (mixed papilloma). Mixed papilloma is an extremely rare, benign pulmonary tumor with a typical papillary appearance, showing squamous and glandular epithelial differentiation. The histological and immunohistochemical features of each tumor were analyzed. In addition, molecular pathological examination for epidermal growth factor receptor (EGFR) showed no mutation in two primary cancers. Mixed papilloma showed no BRAF V600E mutation or HPV infection. The present case report provides a clinicopathological understanding of an instance in which three tumors of different pathological types are present in the same lung lobe. Furthermore, it provides a literature review regarding multiple lung nodules, focusing on the clinicopathological diagnosis, clinical treatment, and prognostic assessment of these nodules. This is the first case report of mixed papilloma arising in MPLC.
\end{abstract}

Keywords: multiple primary lung cancer, mixed squamous cell and glandular papilloma, squamous, adenocarcinoma, multiple lung nodules

\section{Introduction}

MPLC refers to the discovery of two or more primary lung cancers simultaneously or successively in the same or bilateral lobes. It is categorized as either synchronous multiple primary lung cancer (sMPLC) or metachronous multiple primary lung cancer (mMPLC), based on the time interval. The incidence rate of MPLC ranges from 0.2 to $20 \%{ }^{1}$ with no significant difference in morbidity based on sex, and most patients diagnosed were found to have two nodules. ${ }^{2-5}$ Moreover, with recent developments in imaging technology, the annual rate of multiple pulmonary nodules detection has increased but the diagnosis and management are still confusing. ${ }^{6}$

Solitary endobronchial papilloma account for $0.38 \%$ of all lung tumors. ${ }^{7}$ A solitary endobronchial papilloma is divided into three subtypes based on morphological features: squamous cell papilloma, glandular papilloma, and mixed papilloma. Mixed papilloma is the least common subtype and consists of 
bidirectionally differentiated squamous and glandular epithelial cells. According to previous research, mixed papilloma tumors are usually central and endobronchial or, in rare cases, peripheral. Patients usually lack clinical symptoms and are diagnosed on physical examination. Complete surgical resection is the preferred treatment, which results in a good prognosis in most cases. ${ }^{8}$

In recent years, owing to the increase in detection rates, MPLC has attracted increasing attention. This case provides insight into the clinical diagnosis and treatment of MPLC for pathologists and clinicians.

\section{Materials and Methods}

The resected pulmonary samples were paraffinembedded, after which the blocks were cut into 4- $\mu \mathrm{m}$ sections and stained with hematoxylin and eosin. Immunohistochemical examination was performed using the SP kit. The antibodies included CK5/6, p63, TTF-1, P16, and Ki-67. Special staining techniques included Periodic Acid-Schiff (PAS) and Alcian bluePeriodic Acid-Schiff (AB-PAS) stains. Amplification Refractory Mutation System Polymerase chain reaction (ARMS-PCR) was used to detect the expression of BRAF V600E and EGFR (including exons 18, 19, 20, and 21) gene mutations. Flow-through hybridization was done to determine the genotypic variants of the human papilloma virus (HPV).

\section{Clinical Summary}

In August 2019, on physical examination, a 74-year-old man was found to have two partial solid nodules and a ground-glass opacity (GGO) in the upper lobe of his right lung. He had no respiratory symptoms, though he had smoked 20 cigarettes per day for 30 years. The diameter of the maximum nodule was $0.9 \mathrm{~cm}$, and a follow-up was recommended. In July 2020, computed tomography (CT) showed nodules grown. The nodule (labeled tumor A) at the apex of the right upper lobe had accreted from $0.5 \mathrm{~cm}$ to $1 \mathrm{~cm}$ in diameter and its density increased. CT displayed tumor A with spiculated margins, closed to the pleura and bronchioles near the tumor were tugged and dilated. The second tumor (labeled tumor B), a pure GGO with a maximum diameter about $0.7 \mathrm{~cm}$, did not change significantly from 11 months ago. The third tumor (labeled tumor C) was in the anterior segment of the lung and had an irregular shape but well defined. Its diameter accreted from $0.9 \mathrm{~cm}$ to $1.2 \mathrm{~cm}$, accompanied by an increase in density (Figure 1).

Owing to the patient's poor lung function and his refusal to consent to a lobectomy, a video-assisted thoracic surgery segmentectomy was performed to resect lesions on July 6, 2020. Additionally, the mediastinal lymph nodes, including groups 2, 4, 7, 10 and 11, were resected for disease staging. Intraoperatively, the three nodules were found to be isolated from each other, and all three were completely resected, with at least $2 \mathrm{~cm}$ between the lesions and the cutting edge. No metastatic lymph nodes were observed. The patient did not receive chemoradiotherapy after the surgery, and at the 16month follow-up, no recurrence or metastasis was found.
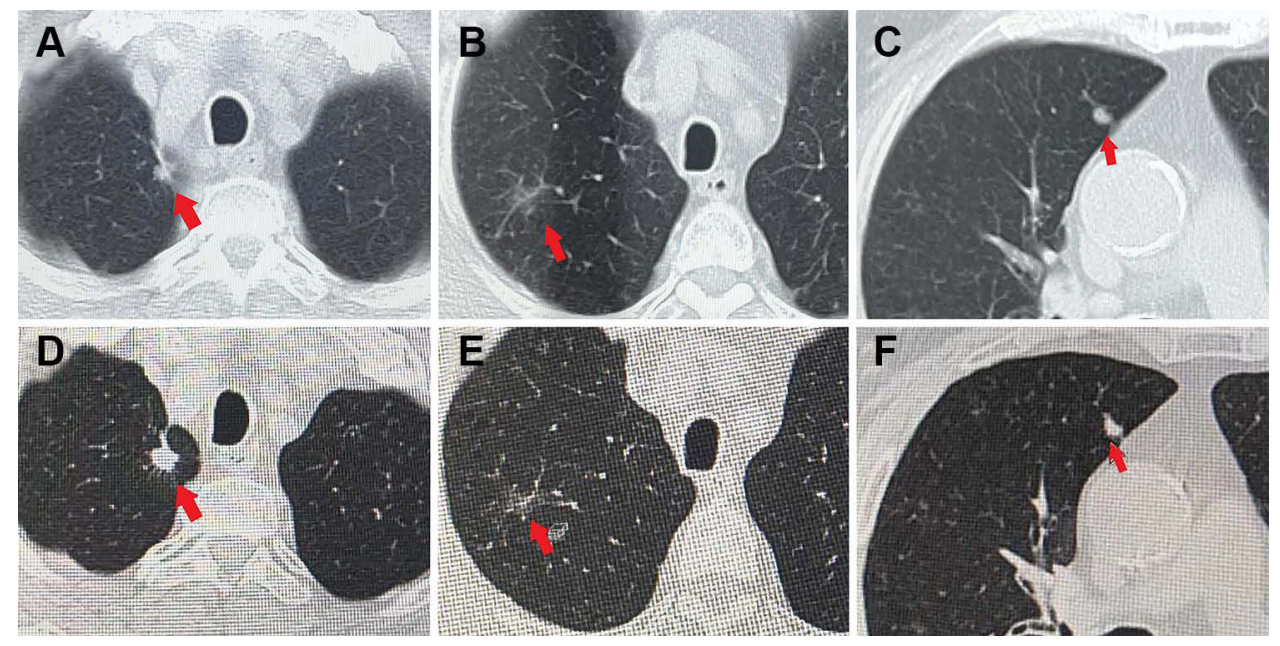

Figure I In August 2019, three nodules were found during physical examination. (A-C) shown tumor A, tumor B and tumor C, respectively; In July 2020, increased nodular diameter and density were observed at follow-up. (D-F) shown tumor A, tumor B and tumor C, respectively. The red arrows show pulmonary nodules. 


\section{Results: Tumor A: Macroscopy, Histopathology, Immunohistochemistry}

The lesion was white, slightly stiffer in texture and the boundary between the tumor and the surrounding lung tissue was unclear. A frozen section was prepared intraoperatively and examination of the frozen section indicated SCC. Postoperatively, the tumor specimen was embedded in a paraffin block and examined.

Under the microscope, a maximum diameter of approximately $0.8 \mathrm{~cm}$ was observed. Histological examination of tumor A revealed a moderately differentiated SCC with cytoplasmic keratosis and no keratin pearl. Carcinomas formed irregular nests and strands of tumor cells separated by varying amounts of fibrous stroma. The mitotic figures and necrosis were common. Small pulmonary alveoli were observed around the tumor cells, indicating that the tumor was located at the periphery of the lung. Some round or irregular gland-like or tubular structures, regarded as alveolar architectures, were observed in the cancer cell nests. Immunostaining analysis indicated that the cancer cells were positive for the expression of $\mathrm{p} 63$ and CK $5 / 6$ but negative for the expression of TTF-1, whereas the alveolar architecture among the cancer cells was positive for the expression of TTF-1 (Figure 2). The Ki-67 proliferation index was $50 \%$. Based on these observations, a diagnosis of stage IA1 peripheral SCC with (T1aN0M0) was confirmed.

\section{Tumor B: Macroscopy, Histopathology, Immunohistochemistry}

Tumor B was grayish white in color, with medium texture and well defined. It was $2 \mathrm{~cm}$ from tumor $\mathrm{A}$, when observed intraoperatively. An intraoperative pathological examination of the frozen section indicated squamous cell papilloma with mild dysplasia.

Under the microscope, tumor B was composed of branching papillary structures with an axis of fibroblasts in a mucinous background. The papilla had various forms, with broad and slender branches visible, and was composed of both squamous and glandular cells (Figure 3). The tumor cells were neatly arranged with an obvious polar direction and no atypia. The squamous epithelium was lined with squamous cells that were positive for the expressions of CK5/6 and P63. The glandular epithelium was lined with pseudostratified ciliated and non-ciliated columnar cells with scattered mucinfilled cells, which were negative for the expressions of both p63 and CK 5/6. Both components were focally positive for
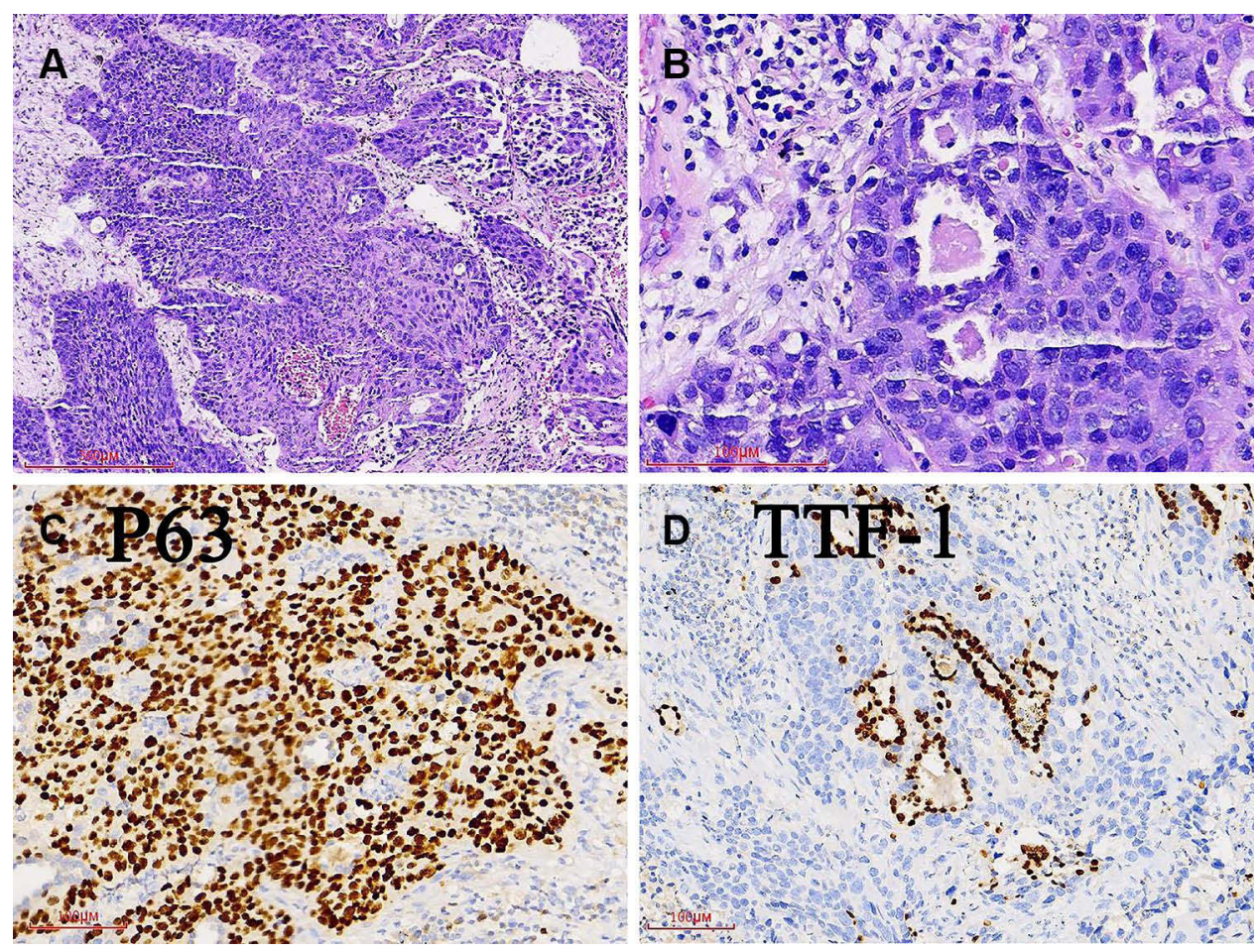

Figure 2 Cancer cells of tumor A were arranged in irregular nests (A). Alveolar architectures were preserved in cancer tissues (B). Immunohistochemical staining of tumor cells were positive for p63 (C) but negative for TTF-I (D). 

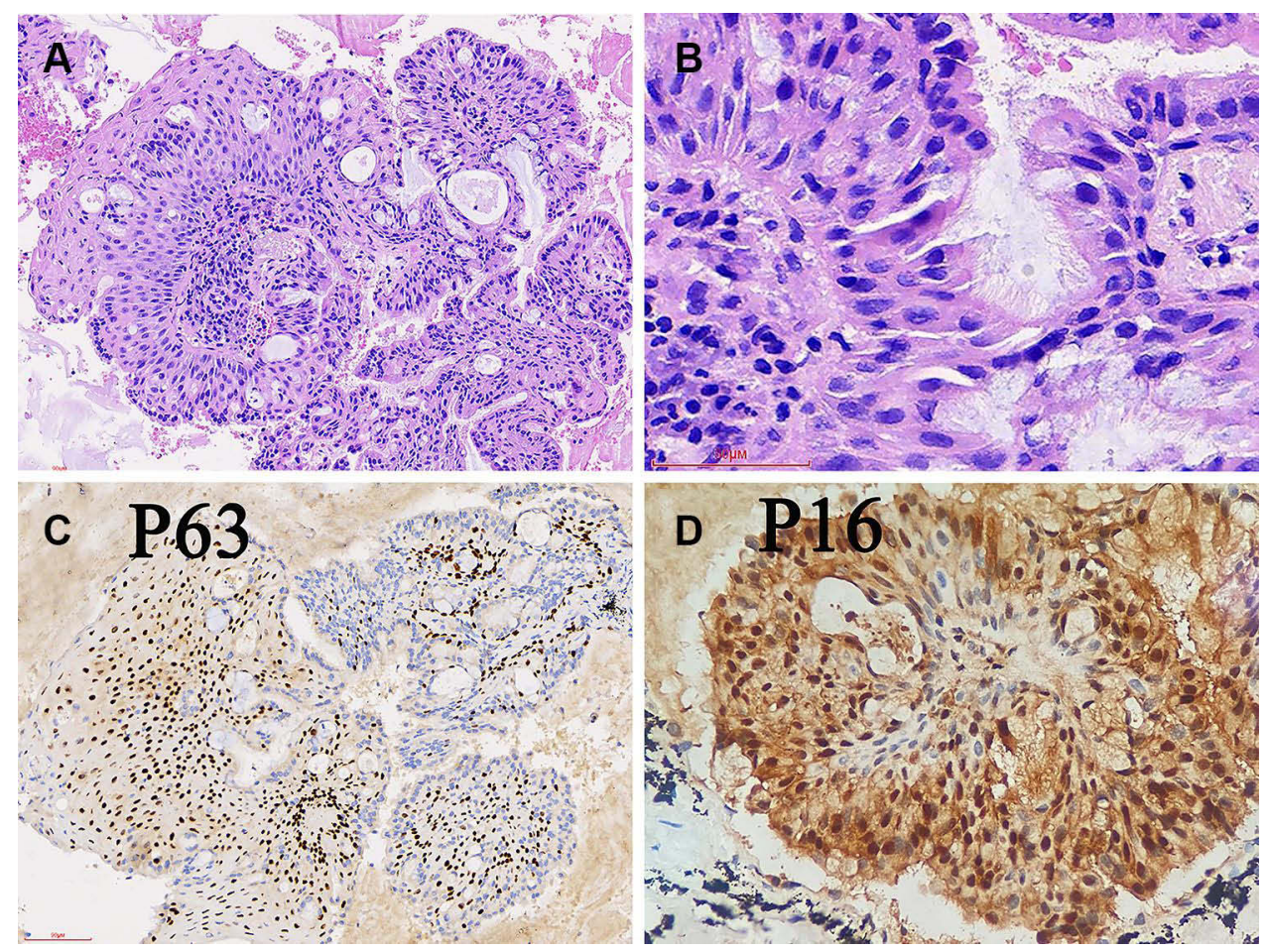

Figure 3 (A) Tumor B consisted of branching papillary structures with an axis of fibroblasts, showing a mixture of squamous and glandular cells; (B) Pseudostratified ciliated columnar tumor cells; (C) Immunostaining showing that some tumor cells are positive for the expression of p63; (D) Both components were focally positive for PI6.

P16 and negative for TTF-1. The Ki-67 proliferation index was $3 \%$, and AB-PAS staining showed various dark and light blue mucus components in- and outside the cells. PAS staining showed positive expression of glandular components. Based on these observations, a diagnosis of mixed squamous cell and glandular papilloma was confirmed.

\section{Tumor C: Macroscopy, Histopathology, Immunohistochemistry}

Tumor $\mathrm{C}$ was whitish gray in color, with ill-defined borders. The intraoperative examination of the frozen section revealed an adenocarcinoma. Microscopically, the maximum diameter of the tumor was $1.2 \mathrm{~cm}$, and the maximum diameter of the invasive portion was approximately $0.7 \mathrm{~cm}$. The cancer cells were well differentiated. The central part of the nodule, which comprised $60 \%$ of the tumor, was an invasive acinar cell carcinoma (Figure 4), and the surrounding lepidic structure accounted for the remaining $40 \%$. On immunohistochemical analysis, the tumor cells were found to be positive for the expression of TTF-1 and negative for the expression of CK5/6. Scattered P63-positive cells were detected in

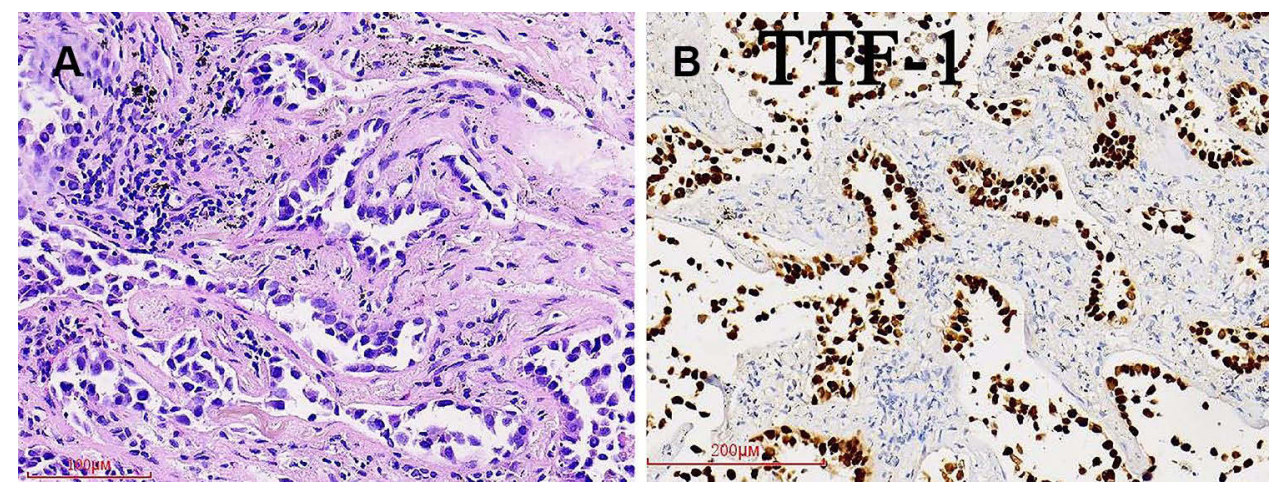

Figure 4 (A) Tumor $C$ was well differentiated. The central part of the nodule, which comprised $60 \%$ of the tumor, was an invasive acinar cell carcinoma; (B) Immunostaining showing that tumor cells are positive for the expression of TTF-I. 

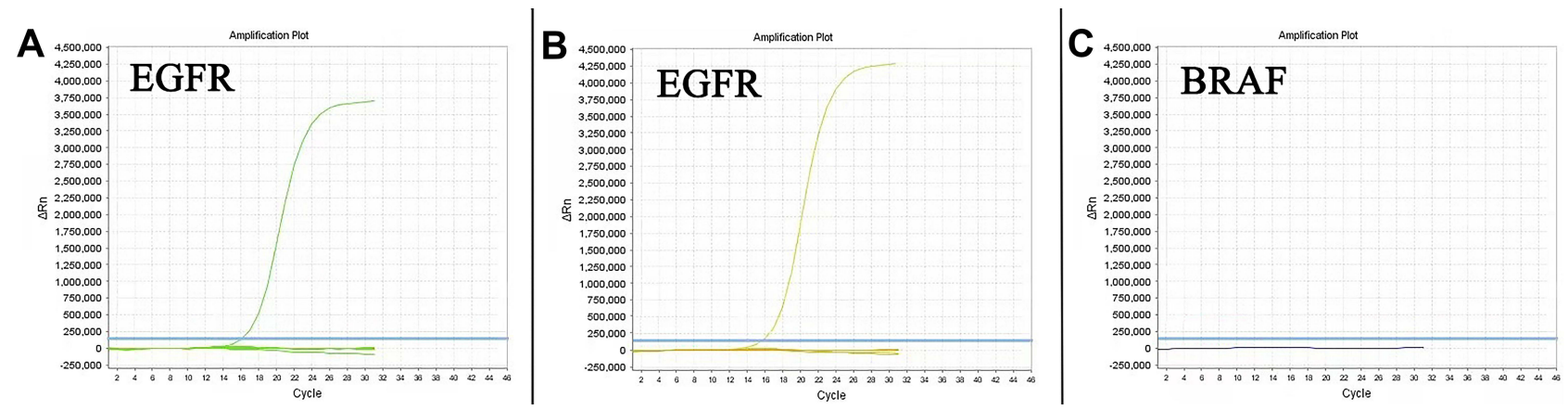

Figure 5 Molecular pathological examination for EGFR showed no mutation in squamous cell carcinoma (A) and adenocarcinoma (B). Mixed papilloma had no BRAF V600E mutation (C).

immunostaining, and the Ki-67 proliferation index was $10 \%$. Based on these observations, a diagnosis of stage IA2 invasive adenocarcinoma with (T1bN0M0) was confirmed.

\section{Molecular Pathological Examination}

Molecular pathological examination for EGFR showed no mutation in SCC and adenocarcinoma. Mixed papilloma had no BRAF V600E mutation or HPV infection (Figure 5).

\section{Discussion}

With the increasing incidence of lung cancer, the detection rate of MPLCs has gradually increased in recent years. ${ }^{9}$ More than half of all MPLC patients have two nodules. Adenocarcinoma accounts for the largest portion, among which acinar, lepidic, and papillary adenocarcinomas are the three most frequent architectural patterns. ${ }^{10,11}$ In 1975, Martini and Melamed proposed the first clinicopathological diagnostic criteria for MPLC. ${ }^{3}$ This standard has been widely accepted by clinicians and pathologists, and has been an important diagnostic reference for many years. However, the limitations of this standard became obvious as only the major histological tumor types (adenocarcinoma and SCC) common then were taken into consideration, whereas it is now known that adenocarcinomas have significant morphological heterogeneity and that adenosquamous carcinomas also exist. The American College of Chest Physicians (ACCP) revised the diagnostic standard in 2003, 2007, and 2013, and combined the comprehensive histologic criteria from Martini and Melamed with the evaluation of morphology, immunohistochemistry, and molecular features. ${ }^{12}$ Some researchers suggested that a comprehensive histologic assessment, including the evaluation of the percentages of histologic subtypes, histologic features, stromal characteristics, lymphoid hyperplasia, and/or necrosis, supersedes the clinical criteria described by Martini and Melamed in differentiating multiple primary tumors from metastases. ${ }^{13}$ While MPLC and intrapulmonary metastases have somewhat different clinical presentations, reliably differentiating pathologically between two nodules with the same histological type remains an important challenge for clinicians and pathologists.

The pathological TNM stage of multiple pulmonary nodules is also challenging due to the difficulty in diagnosing MPLC correctly. The 8th edition of the American Joint Committee on Cancer (AJCC) staging manual divides patients with multiple pulmonary nodules into four categories: MPLC, intrapulmonary metastases, multifocal ground-glass/lepidic adenocarcinoma, and pneumonic-type adenocarcinoma. ${ }^{14}$ MPLC comprising two or more distinct and histologically different masses are considered unrelated, and TNM staging alone has been recommended for each tumor. Considering the histological heterogeneity of adenocarcinomas, it is necessary to use comprehensive histologic subtyping to characterize the histologic features.

For most physicians, surgery is the preferred treatment for MPLC as the five-year survival rate after the complete resection of MPLC is approximate to that of single lung cancers. ${ }^{10,15}$ The scope of surgery includes lobectomy and sub-lobar resection (including segmentectomy and wedge resection). Some surgeons prefer lobectomy considering lower postoperative local recurrence rate. ${ }^{16-18}$ Some previous studies have indicated that there was no significant difference in prognosis between lobectomy and segmentectomy. ${ }^{19,20}$ Segmentectomy was considered to be the first choice in patients with clinical stage IA non-small cell lung cancer (NSCLC), especially in older patients who 
have underlying comorbidities such as cerebrovascular accident. $^{2,21}$ The present case study was of an older patient with emphysema and multiple chronic underlying diseases. A total of three nodules were in different areas of the upper lobe of the right lung. During preoperative communication, the patient and his family members rejected the right lung lobectomy procedure and opted for a segmentectomy, to retain more normal lung tissue. To completely remove the tumor and protect respiratory function, thoracoscopic partial resection of the upper lobe of the right lung was performed, with a safe margin of more than $2 \mathrm{~cm}$.

EGFR is a transmembrane cell surface receptor implicated in signal transduction pathways that regulate cell proliferation and apoptosis. Approximately 50\% of Asians have EGFR-driven NSCLC ${ }^{22}$ and EGFR mutation is important for the selection of targeted therapy and the development of resistance. In this case, molecular pathological examination for EGFR showed no mutation in SCC and adenocarcinoma.

Mixed papilloma is rare and only sporadic cases have been documented. Mixed papilloma is predominantly lesion of male smokers, and the median age is sixth decade. $^{23}$ It occurs most commonly in the central tracheobronchial tree. ${ }^{24}$ In contrast to squamous cell papilloma which has a strong association with HPV, all the reported cases of mixed papilloma are HPVnegative. $^{23,25}$ In this case, we cut 5-micron rolls from paraffin-embedded block and extracted DNA for PCR amplification. And then flow-through hybridization was done to determine the HPV DNA. Although there was usually some loss of DNA during the process of making wax blocks in lung specimens, remaining HPV DNA could be detected as this technique had the characteristics of high sensitivity and specificity. Consistent with previous reports, our test showed mixed papilloma without HPV infection. Mixed papilloma had frequent BRAF V600E mutations. ${ }^{25,26}$ Differently, we found no BRAF mutation in this case. Data ${ }^{23,24}$ demonstrated malignant transform could occur in some mixed papilloma. In our case, no cellular atypia was found in the mixed papilloma and the three well differentiated nodules were isolated from each other, occurred at similar time. Therefore, SCC and adenocarcinoma in our case were primary rather than development of cancer in mixed papilloma. It is also worth to note that in peripheral lung cancer, alveoli are occasionally seen spotted in cancerous tissue, which can be misdiagnosed as adenocarcinoma or carcinoma with glandular differentiation. ${ }^{27}$
In this case, immunohistochemistry was instrumental in differentiating normal alveoli from adenocarcinoma.

In conclusion, the diagnosis and treatment of MPLC remain major problems that should be addressed by collecting relevant data and making a comprehensive decision. With the increase in confirmed cases, the accumulation of more clinicopathological data would be helpful to reach a consensus on the ideal clinical diagnosis and treatment protocols.

\section{Abbreviations}

MPLC, multiple primary lung cancer; SCC, squamous cell carcinomas; EGFR, epidermal growth factor receptor; sMPLC, synchronous multiple primary lung cancer; mMPLC, metachronous multiple primary lung cancer; PAS, Periodic Acid-Schiff; AB-PAS, Alcian blue-Periodic Acid-Schiff; PCR, polymerase chain reaction; ARMS-PCR, Amplification Refractory Mutation System PCR; HPV, human papilloma virus; GGO, ground-glass opacity; CT, computed tomography; ACCP, American College of Chest Physicians; AJCC, American Joint Committee on Cancer; NSCLC, non-small cell lung cancer.

\section{Ethical Approval}

Institutional approval was not required to publish the case details.

\section{Consent for Publication}

Written informed consent was obtained from the patient for publication of this paper and any accompanying images.

\section{Acknowledgment}

This work was supported by the Project funded by Natural Science Foundation of Liaoning Province (No.2021 -BS106, Di Zhang) and National Natural Science Foundation of China (No. 81472599, Chuifeng Fan).

\section{Disclosure}

The authors report no conflicts of interest in this work.

\section{References}

1. Murphy SJ, Aubry MC, Harris FR, et al. Identification of independent primary tumors and intrapulmonary metastases using DNA rearrangements in non-small-cell lung cancer. $J$ Clin Oncol. 2014;32:4050-4058. doi:10.1200/JCO.2014.56.7644

2. Jung EJ, Lee JH, Jeon K, et al. Treatment outcomes for patients with synchronous multiple primary non-small cell lung cancer. Lung Cancer. 2011;73:237-242. doi:10.1016/j.lungcan.2010.11.008 
3. Martini N, Melamed MR. Multiple primary lung cancers. $J$ Thorac Cardiovasc Surg. 1975;70:606-612. doi:10.1016/S0022-5223(19) 40289-4

4. Vazquez M, Carter D, Brambilla E, et al. Solitary and multiple resected adenocarcinomas after $\mathrm{CT}$ screening for lung cancer: histopathologic features and their prognostic implications. Lung Cancer. 2009;64:148-154. doi:10.1016/j.lungcan.2008.08.009

5. Dai L, Yang HL, Yan WP, et al. The equivalent efficacy of multiple operations for multiple primary lung cancer and a single operation for single primary lung cancer. $J$ Thorac Dis. 2016;8:855-861. doi:10.21037/jtd.2016.03.42

6. Jiang L, He J, Shi X, et al. Prognosis of synchronous and metachronous multiple primary lung cancers: systematic review and meta-analysis. Lung Cancer. 2015;87:303-310.

7. Inamura K, Kumasaka T, Furuta R, et al. Mixed squamous cell and glandular papilloma of the lung: a case study and literature review. Pathol Int. 2011;61:252-258.

8. Li F, He M, Li F, et al. Histologic characteristics and prognosis of lung mixed squamous cell and glandular papilloma: six case reports. Int J Clin Exp Pathol. 2019;12:3542-3548.

9. Ishikawa Y, Nakayama H, Ito H, et al. Surgical treatment for synchronous primary lung adenocarcinomas. Ann Thorac Surg. 2014;98:1983-1988. doi:10.1016/j.athoracsur.2014.07.006

10. Tie H, Luo J, Shi R, et al. Characteristics and prognosis of synchronous multiple primary lung cancer after surgical treatment: a systematic review and meta-analysis of current evidence. Cancer Med. 2020;10:507-520. doi:10.1002/cam4.3614

11. Yu YC, Hsu PK, Yeh YC, et al. Surgical results of synchronous multiple primary lung cancers: similar to the stage-matched solitary primary lung cancers? Ann Thorac Surg. 2013;96:1966-1974. doi:10.1016/j.athoracsur.2013.04.142

12. Detterbeck FC, Mazzone PJ, Naidich DP, et al. Screening for lung cancer: diagnosis and management of lung cancer, 3rd ed: American College of Chest Physicians evidence-based clinical practice guidelines. Chest. 2013;143:e78S-e92S. doi:10.1378/chest.12-2350

13. Girard N, Deshpande C, Lau C, et al. Comprehensive histologic assessment helps to differentiate multiple lung primary nonsmall cell carcinomas from metastases. Am $J$ Surg Pathol. 2009;33:1752-1764. doi:10.1097/PAS.0b013e3181b8cf03

14. Schneider F, Dacic S. Histopathologic and molecular approach to staging of multiple lung nodules. Transl Lung Cancer Res. 2017;6:540-549. doi:10.21037/tlcr.2017.06.11

15. Chen TF, Xie CY, Rao BY, et al. Surgical treatment to multiple primary lung cancer patients: a systematic review and meta-analysis. BMC Surg. 2019;19:185. doi:10.1186/s12893-019-0643-0

16. Zuin A, Andriolo LG, Marulli G, et al. Is lobectomy really more effective than sublobar resection in the surgical treatment of second primary lung cancer? Eur J Cardiothorac Surg. 2013;44:e120-e125. doi:10.1093/ejcts/ezt219
17. Subramanian M, McMurry T, Meyers BF, et al. Long-term results for clinical stage IA lung cancer: comparing lobectomy and sublobar resection. Ann Thorac Surg. 2018;106:375-381. doi:10.1016/j. athoracsur.2018.02.049

18. Whitson BA, Groth SS, Andrade RS, et al. Survival after lobectomy versus segmentectomy for stage I non-small cell lung cancer: a population-based analysis. Ann Thorac Surg. 2011;92:1943-1950. doi:10.1016/j.athoracsur.2011.05.091

19. Zhang Z, Gao S, Mao Y, et al. Surgical outcomes of synchronous multiple primary non-small cell lung cancers. Sci Rep. 2016;6:23252. doi:10.1038/srep23252

20. Shah AA, Barfield ME, Kelsey CR, et al. Outcomes after surgical management of synchronous bilateral primary lung cancers. Ann Thorac Surg. 2012;93:1055-1060. doi:10.1016/j.athoracsur.2011.12. 070

21. Koike T, Koike T, Yoshiya K, et al. Risk factor analysis of locoregional recurrence after sublobar resection in patients with clinical stage IA non-small cell lung cancer. $J$ Thorac Cardiovasc Surg. 2013;146:372-378. doi:10.1016/j.jtcvs.2013.02.057

22. Travis WD, Brambilla E, Nicholson AG, et al. The 2015 World Health Organization classification of lung tumors: impact of genetic, clinical and radiologic advances since the 2004 classification. $J$ Thorac Oncol. 2015;10(9):1243-1260. doi:10.1097/JTO.000000 0000000630

23. Flieder DB, Koss MN, Nicholson A, et al. Solitary pulmonary papillomas in adults: a clinicopathologic and in situ hybridization study of 14 cases combined with 27 cases in the literature. Am J Surg Pathol. 1998;22(11):1328-1342. doi:10.1097/00000478-199811000-00003

24. Tryfon S, Dramba V, Zoglopitis F, et al. Solitary papillomas of the lower airways: epidemiological, clinical, and therapeutic data during a 22-year period and review of the literature. $J$ Thorac Oncol. 2012;7 (4):643-648. doi:10.1097/JTO.0b013e3182468d06

25. Huang YL, Chang YL, Chen KC, et al. Mixed squamous cell and glandular papilloma of the lung: a case report of a novel mutation in the BRAF gene and coexistent HPV infection, possible relationship to ciliated muconodular papillary tumor. Pathol Int. 2019;69(2):1 04-109. doi:10.1111/pin.12747

26. Sasaki E, Masago K, Fujita S, et al. AKT1 mutations in peripheral bronchiolar papilloma: glandular papilloma and mixed squamous cell and glandular papilloma is distinct from bronchiolar adenoma. $A m$ $J$ Surg Pathol. 2021;45(1):119-126. doi:10.1097/PAS.000000000 0001573

27. Fan X, Zhang X, Wang E, et al. Alveolar architectures preserved in cancer tissues may be potential pitfalls for diagnosis and histological subtyping of lung cancer: three case reports. Medicine. 2018;97: e12613. doi:10.1097/MD.0000000000012613
OncoTargets and Therapy

\section{Publish your work in this journal}

OncoTargets and Therapy is an international, peer-reviewed, open access journal focusing on the pathological basis of all cancers, potential targets for therapy and treatment protocols employed to improve the management of cancer patients. The journal also focuses on the impact of management programs and new therapeutic

Submit your manuscript here: https://www.dovepress.com/oncotargets-and-therapy-journa agents and protocols on patient perspectives such as quality of life, adherence and satisfaction. The manuscript management system is completely online and includes a very quick and fair peer-review system, which is all easy to use. Visit http://www.dovepress.com/ testimonials.php to read real quotes from published authors. 